Methods Qualitative interview study with adults living with UC recruited from three Inflammatory Bowel Disease (IBD) services in the North of England, undertaken between 4th June and 31st October 2019. Data were collected during telephone interviews, digitally recorded and transcribed. Inductive thematic analysis was performed by two researchers using NVivo software. Codes were cross-checked and data saturation was confirmed prior to study close.

Results A total of 33 adults participated (51\% female, median age 39 years, median time since diagnosis 6 years). Thematic saturation was confirmed. Four key themes were identified in the data. (1) Treatment effectiveness: this was the primary concern of all participants when choosing a new treatment. Participants explained that alleviating symptoms improving quality of life was the most important driver of their treatment preferences. (2) Influence of healthcare professionals: treatment discussions and choices were heavily guided by IBD healthcare professionals (HCPs). Most participants in this study described the valuable relationships that they have within IBD nurses and medics, and how they trust and respect their clinical expertise. (3) Other influences: whilst important to treatment choices, participants placed limited value on the route of administration and side effects relative to treatment effectiveness overall. (4) Changes over time: there was an increased willingness to try alternative treatments, and eventually surgery over time, in accordance with the severity and duration of symptoms, and crucially, as medical treatment options are exhausted.

Conclusion The importance of treatment efficacy and the influential role of HCPs when patients choose treatments for steroid resistant UC has been highlighted - with a willingness to consider different treatments over time. Less value was placed on side-effects and route of administration. This study provides a qualitative perspective on patient preferences which should be considered in practice guidelines and trial design.

\section{P97 MANAGEMENT OF STEROID RESISTANT ULCERATIVE COLITIS - A NATIONAL SURVEY OF UK PRACTICE}

${ }^{1} \mathrm{E}$ Coates*, ${ }^{1} \mathrm{~A}$ Barr, ${ }^{1} \mathrm{~N}$ Totton, ${ }^{1} \mathrm{D}$ Hind, ${ }^{1} \mathrm{P}$ Shackley, ${ }^{2} \mathrm{~S}$ Blackwell, ${ }^{2} \mathrm{~N}$ Dames, ${ }^{2} \mathrm{H}$ Bedford, ${ }^{1,3} \mathrm{M}$ Lee, ${ }^{4} \mathrm{~S}$ Sebastian, ${ }^{5} \mathrm{C}$ Probert, ${ }^{3} \mathrm{~A}$ Lobo. ${ }^{1}$ University of Sheffield, Sheffield, UK; ${ }^{2}$ Patient Representative; ${ }^{3}$ Sheffield Teaching Hospitals NHS Foundation Trust, Sheffield, UK; ${ }^{4}$ Hull University Teaching Hospitals NHS Trust, Hull, UK; ${ }^{5}$ University of Liverpool, Liverpool, UK

\subsection{6/gutjnl-2020-bsgcampus. 172}

Introduction Corticosteroids are a mainstay in the treatment of moderately severe relapses of ulcerative colitis (UC), yet almost $50 \%$ of patients do not respond fully, with the risk of prolonged steroid use and side-effects. There is insufficient evidence to inform optimum treatment choice for steroid resistant disease. The aim of this study was to provide details of current practice in the management of steroid resistant UC. Methods A cross-sectional survey of Inflammatory Bowel Disease (IBD) healthcare professionals (HCPs) in the UK was conducted online using the Qualtrics platform. HCPs were invited to participate in the survey through professional networks: British Society of Gastroenterology IBD section, Royal College of Nursing IBD Nurses Network, and social media. Clinical scenarios representing patients with moderately severe UC with continuing symptoms (steroid resistant (SR)) and with relapse after steroid dose reduction (steroid dependent (SD)) were included - both thiopurine treated $(\mathrm{TP}+)$ and naïve (TP-
). Data were analysed descriptively with chi-squared tests on outcomes of interest using $\mathrm{R}$ software.

Results 387 HCPs visited the survey; 47\% (168 HCPs) consented (68\% medical; 30\% nurses; median 7.5 years since appointment) across all UK regions. Definitions of steroid resistance varied: $68 \%$ indicating an incomplete response to prednisolone $40 \mathrm{mg} /$ day after 2 weeks and after 4 weeks in a further 58\%. Only $13 \%$ felt that SR and SD disease should be treated identically.

Anti-TNF drugs would be most frequently offered in each scenario (SR: TP+ 95\%; TP- 87\%; SD: TP+ 88\%; TP- 74\%) with infliximab most frequently suggested; apart from SD TPpatients flaring at prednisolone $5 \mathrm{mg} /$ day. Admission for IV steroids was offered more often for SR disease (32\%) than for SD (12\%).

In SD scenarios, thiopurine treatment would be instigated in TP- patients flaring at $25 \mathrm{mg}$ prednisolone or $5 \mathrm{mg} /$ day in $49 \%$ and in $70 \%$ respectively. Anti-TNF treatment would be offered more frequently if patients are $\mathrm{TP}+$ in both SR (TP+ $81 \%$; TP- $62 \% \mathrm{p}=0.004$ ) or SD patients and particularly for those relapsing at $5 \mathrm{mg} /$ day (Relapse at $25 \mathrm{mg} /$ day: $\mathrm{TP}+78 \%$ TP- 49\%, p $<0.001$; Relapse at $5 \mathrm{mg} /$ day TP $+85 \%$ TP- 46\%, $\mathrm{p}<0.001)$. For both SR and SD disease, $43 \%$ and $58 \%$ respectively felt that endoscopy is not warranted.

Conclusions There are important variations in practice in the UK in how to define, treat and use endoscopy in steroid resistant UC. Such variations need to be understood as part of initiatives to change practice - particularly to avoid excess steroid use - and in trial design.

\section{\begin{tabular}{l|l} 
P98 & DISEASE INVOLUTION IS COMMON AMONG
\end{tabular} PAEDIATRIC CROHN'S DISEASE PATIENTS: A STUDY FROM THE BIOLOGIC ERA}

${ }^{1}$ Mike Davies* ${ }^{2}$ Sreedhar Subramanian, ${ }^{3}$ Christos Tzivinikos, ${ }^{3}$ Marcus Karl-Heinz Auth ${ }^{3}$ Jeng Cheng, ${ }^{2}$ Philip J Smith, ${ }^{2}$ Paul Collins, ${ }^{3}$ Anastasia Konidari, ${ }^{2}$ Andrew Ross, ${ }^{2}$ Bruno Gnaneswaran, ${ }^{2}$ Morwenna Coultate, ${ }^{2}$ George Pears, ${ }^{4}$ Susanna Dodd. ${ }^{1}$ Countess Of Chester Hospital, Chester, UK; ${ }^{2}$ Royal Liverpool University Hospital, Liverpool, UK; ${ }^{3}$ Alder Hey Children's NHS Foundation Trust, Liverpool, UK; ${ }^{4}$ University of Liverpool, UK

\subsection{6/gutjnl-2020-bsgcampus.173}

Introduction Paediatric Crohn's disease (PCD) often presents with a pan-enteric phenotype at diagnosis. However, its longterm evolution in to adulthood, especially since the advent of biological therapy, is not well characterised. Only few studies have assessed this change, with conflicting results and limited by short follow-up times. Our study aimed to evaluate how the PCD phenotype evolves through childhood into adult life.

Methods We performed a single-centre retrospective study of PCD patients diagnosed $\leq 16$ years of age, transitioned to an adult gastroenterology unit with a minimum follow up of 2 years. Disease location and behaviour was characterised using Paris and Montreal classification at diagnosis and follow-up respectively. We assessed for evidence of disease extension or involution as well as variables associated with complicated disease behaviour and surgery. Comparison of frequencies was performed using Pearson's chi-square test. Hazard ratios from Cox proportional hazards models were used to quantify risk of surgery and complicated disease behaviour.

Results 132 patients were included, transitioning to adult care between 2002 and 2016. The median age at diagnosis was 13 (IQR 11-14) and median follow up 11 years. At diagnosis, 27 
(20.4\%) patients had complicated disease behaviour compared to $46(34.9 \%)$ at follow up $(\mathrm{p}=0.0018) .83(62.9 \%)$ patients had a 'pan-enteric' phenotype at diagnosis, however only 55 $(66.3 \%)$ retained this phenotype at follow-up $(p=0.0002)$. Disease extension was noted in $18.9 \%$ of patients and involution in $35.6 \%$ of patients, with upper GI disease noted in only $15.9 \%$ of patients at follow-up $(\mathrm{p}=0.0001)$. There was a high exposure to both thiopurines (91.7\%) and biologics (63.6\%), with a median time to starting treatment of 0 (IQR $0-1$ ) and 5 (IQR 2-7) years for thiopurines and anti-TNF therapy respectively. The rate of exposure to biological therapy was similar in patients with disease involution $(32 / 47,68.1 \%)$ and disease extension $(21 / 25,84 \%)$. The cumulative probability $(95 \% \mathrm{CI})$ of surgery was $0.05(0.02,0.11)$ at 1 year, 0.17 $(0.11,0.24)$ at 3 years and $0.22(0.15,0.30)$ at 5 years respectively. Overall, $56(42.4 \%)$ patients had surgery at the end of follow-up. Neither disease location nor behaviour were associated with need for surgery.

Conclusions Changes in both disease location and behaviour were seen in our PCD cohort as they progressed to adult life. A significant proportion had disease involution, likely related to a high rate of exposure to biological therapy.

\section{P99 ROUTINE IMAGING OF CROHN'S DISEASE WITH ULTRASOUND AND MR ENTEROGRAPHY: THE PATIENT PERSPECTIVE}

Katie Devlin*, Gurinder Nandra, James Pilcher, Richard Pollok, Jaymin Harivadan Patel. St George's University Hospital, London, UK

\subsection{6/gutjnl-2020-bsgcampus. 174}

Introduction Assess patient understanding, tolerance and preferences with regards to modern imaging techniques of small bowel Crohn's disease.

Methods Consecutive paper-based questionnaires were obtained from patients referred for diagnosis, assessment or reassessment of Crohn's disease with MR enterography (MRE) $(n=50)$ and small bowel ultrasound (SBUS) $(n=50)$ at a large teaching hospital. Analysis performed with SPSS as unpaired non-parametric data.

Results 66\% ( $\mathrm{n}=56 / 88)$ of patients were unsure which examination was more 'accurate'. There was an inclination towards preferencing the examination that patient had attended for i.e. the SBUS cohort thought that SBUS was more accurate than MRE (18\% versus 9\%) whilst the MRE group favoured MRE over SBUS (33\% versus 2\%).

Free text responses regarding the cause of discomfort during MRE included: positioning (lying prone), claustrophobia and the volume of oral contrast. 94\% $(n=47)$ of MRE patients received oral contrast vs. $10 \% \quad(n=5)$ of SBUS patients. Responses in the SBUS group related to probe pressure.

Regarding quality of information provided following the test, the mean score was 10 for SBUS patients versus 0 for MRE $(0=$ not given opportunity to speak to someone, $10=$ indicating most satisfied with quality of information provided). Free text responses commented specifically on the ability to see and understand their disease with SBUS.

$60 \%(n=29)$ of the SBUS cohort scored 10 for overall experience (median 10) versus 19\% of the MRE group (median=8). Most participants would undergo the same examination again $(96 \%$ and $100 \%$ of the SBUS and MRE cohorts respectively).

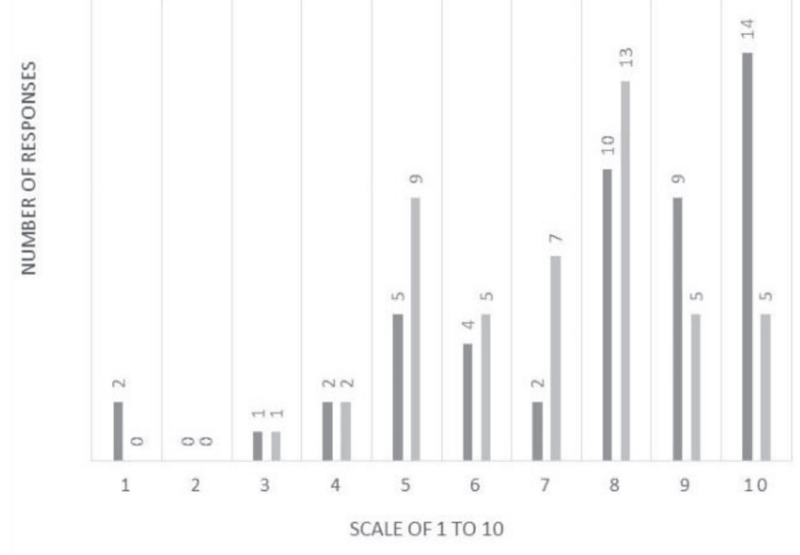

Abstract P99 Figure 1 Patient responses to 'How uncomfortable was the examination? $1=$ Uncomfortable, $10=$ Comfortable

45\% patients had experienced both MRE and SBUS at some point in their history. Of these, 68\% $(n=21)$ preferred SBUS, $16 \%(\mathrm{n}=4)$ preferred MRE and $16 \%$ responded 'either.'

Conclusions SBUS and MRE both demonstrate excellent sensitivity and specificity in the assessment of small bowel inflammation in Crohn's disease. Either examination is suitable as a first-line investigation, although MRE is more sensitive than SBUS in diagnosing the extent of disease. ${ }^{1}$ This study demonstrates that SBUS is often better preferred by patients as a stand-alone test and compared with MRE. This is secondary to a combination of better patient comfort, the ability to immediately discuss findings with a radiologist following the examination and a perceived similar accuracy.

\section{REFERENCES}

1. Taylor SA, Mallett S, Bhatnagar G, et al. Diagnostic accuracy of magnetic resonance enterography and small bowel ultrasound for the extent and activity of newly diagnosed and relapsed Crohn's disease (METRIC): a multicentre trial. Lancet Gastroenterol Hepatol 2018 Aug;3(8):548-558.

\section{P100 OUTCOMES OF A CLINICAL PSYCHOLOGY INTERVENTION IN A UK IBD SERVICE}

Fiona Eldridge*, Konstantina Strongili, Miles Parkes, Tim Raine. Addenbrooke's Hospital, Cambridge University Hospitals NHS Foundation Trust, Cambridge, UK

\subsection{6/gutjnl-2020-bsgcampus. 175}

Introduction Patients with IBD experience significant psychological distress. A variety of interventions to ameliorate this distress have been studied. However, to date no European study has assessed the impact on patients of a clinical psychologist embedded within the IBD team. Clinical psychology is uniquely characterised by its use of a patient/therapist collaborative formulation to guide and select from a variety of evidenced based psychological therapeutic treatment options. Such an approach is likely to be particularly beneficial for patients with IBD who may present with a wide range of needs from adjustment at diagnosis to coping with symptoms, adhering to treatment protocols and managing an on-going relationship with a medical team.

Methods A novel clinical psychology service was established within an existing IBD service at a large UK tertiary centre. Patients were referred either by a gastroenterologist, IBD 